\title{
Approximation of relaxed solutions for lower semicontinuous differential inclusions
}

\author{
by A. Ornelas* (Évora)
}

\begin{abstract}
We construct a guided continuous selection for lsc multifunctions with decomposable values in $L^{1}[0, T]$. We then apply it to obtain a new result on the uniform approximation of relaxed solutions for lsc differential inclusions.
\end{abstract}

Introduction. Let $K$ be a compact metric space. We construct a "guided" continuous selection for multifunctions $G: K \rightarrow L^{1}$ which are lsc (lower semicontinuous) with closed decomposable values contained in a ball. This result refines a selection theorem proved by Fryszkowski [9].

As a consequence of this abstract result we obtain an approximation property for the solution set $S(\xi)$ of a differential inclusion

$$
x^{\prime} \in F(t, x), \quad x(0)=\xi,
$$

where $F: I \times \mathbb{R}^{n} \rightarrow \mathbb{R}^{n}$ is a measurable multifunction which is (at least) lsc in $x$ and has values $F(t, x)$ compact and integrably bounded. A special case of this approximation property is the well known density result of Filippov [8] and Ważewski [19], which says that provided $F(t, x)$ is Lipschitz in $x$ the solution set $S(\xi)$ is dense in the relaxed solution set associated to the convexified problem

$$
x^{\prime} \in \operatorname{co} F(t, x), \quad x(0)=\xi .
$$

This density result was extended by Pianigiani [16] to cover the case in which $F$ has a modulus of continuity relative to $x$ of the Kamke type (i.e. implying uniqueness of solution for differential equations). Tolstonogov-Finogenko [18] extended further this result in order to allow measurable dependence of $F(t, x)$ on $t$. Bressan [4] treated the locally Lipschitz case. However, in all these papers the relationship between the density property and the uniqueness condition is somewhat hidden. In this paper we bring to light

1991 Mathematics Subject Classification: 34A60, 49A50.

* Supported by Instituto Nacional de Investigação Científica, Portugal. 
this relationship, by showing that the density result is a straightforward consequence of the uniqueness condition via the above-mentioned "guided" selection theorem in $L^{1}$ and a uniformly continuous selection theorem in $\mathbb{R}^{n}$ ([15]) applied to $\operatorname{co} F(t, x)$.

In case $F(t, x)$ is just continuous in $x$, a counterexample of Pliś [17] shows that the density property does not hold anymore. However, a weaker approximation property holds. In fact, let $f(t, x)$ be a selection from co $F(t, x)$ which is measurable in $t$ and continuous in $x$. We show in the present paper that there exists a solution $\underline{x}$ of the differential equation $x^{\prime}=f(t, x)$, $x(0)=\xi$, and a sequence $\left(x_{i}\right)$ of solutions to the differential inclusion $x^{\prime} \in F(t, x), x(0)=\xi$, such that the sequence $\left(x_{i}\right)$ converges uniformly to $\underline{x}$. This result was found by Pianigiani [16] under stronger assumptions, namely for $F$ continuous in $(t, x)$ with values $F(t, x)$ contained in a ball of $\mathbb{R}^{n}$.

We use the method of continuous selections in $L^{1}$ which was initiated by Antosiewicz-Cellina [1]. For other applications and refinements of this method see Pianigiani [16], Bressan [3], Fryszkowski [9], Bressan-Colombo [5], Cellina-Marchi [6], Tolstonogov-Finogenko [18] and also [7]. Łojasiewicz jr. [13] also treats the problem (CP) with $F(t, x)$ lsc, but he uses a different method based on polygonal approximate solutions. General information on multifunctions and differential inclusions can be found in [2]. For the history of decomposable sets see Hiai-Umegaki [11], Olech [14] and [5].

After completion of this paper, I have received paper [10] in which a result similar to our Theorem 1 is proved (with different applications).

Assumptions and the selection theorem. Let $I$ be the interval $[0, T]$ and let $K$ be a compact metric space with distance $d$. Denote by $L^{1}$ the space $L^{1}\left(I, \mathbb{R}^{n}\right)$, with norm $|\cdot|_{1}$. A set $D \subset L^{1}$ is said to be decomposable provided the following property holds: whenever $u, v$ are in $D$ and $\chi$ is the characteristic function of a measurable set $S \subset I$ then the function $w:=\chi u+(1-\chi) v$ is also in $D$.

Hypothesis (G). $G: K \rightarrow L^{1}$ is a multifunction and $g_{*}: K \rightarrow L^{1}$ is a function satisfying:

(a) each value $G(u)$ is closed decomposable;

(b) $\exists M: I \rightarrow \mathbb{R}^{+}$integrable such that: $v \in G(u) \Rightarrow|v(t)| \leq M(t)$ a.e.;

(c) $g_{*}(u)(t)$ is in the closed convex hull of $G(u)(t), \forall u \in K$, for a.e. $t$;

(d) $G$ is lsc and $g_{*}$ is continuous.

Theorem 1. Let $G$ and $g_{*}$ satisfy hypothesis $(\mathrm{G})$. Then there exists a sequence $\left(g_{i}\right)$ of continuous selections from the multifunction $G$ such that

$$
\left|\int_{0}^{t}\left[g_{*}(u)-g_{i}(u)\right] d s\right| \leq 1 / i \quad \forall i \in \mathbb{N} \forall t \in I \forall u \in K .
$$


Intermediate results and proofs. Set $L_{+}^{1}:=\left\{\delta \in L^{1}(I, \mathbb{R}): \delta(t) \geq\right.$ 0 a.e.\}.

Proposition 1. Let $\Delta$ be a nonempty bounded decomposable subset of $L_{+}^{1}$. Then there exists a uniquely determined element $\delta_{0}$ in $L_{+}^{1}$ such that:

(i) $\delta \in \Delta \Rightarrow \delta_{0} \leq \delta$ a.e.

(ii) if $\delta_{1} \in L_{+}^{1}$ satisfies " $\delta \in \Delta \Rightarrow \delta_{1} \leq \delta$ a.e." then $\delta_{1} \leq \delta_{0}$ a.e.

Pro of. Follows from Proposition 1 of Bressan-Colombo [5].

For a set $\Delta$ as in Proposition 1, we define Inf $\Delta$ as the unique element $\delta_{0}$ in $L_{+}^{1}$ as stated.

Proposition 2. Fix some element $v$ and some closed bounded decomposable set $V$ in $L^{1}$. Define

$$
\begin{gathered}
D: L^{1} \times L^{1} \rightarrow L_{+}^{1}, \quad D(u, v)(t):=|u(t)-v(t)| \text { a.e. } \\
D(u, V):=\operatorname{Inf}\{D(u, v): v \in V\}, \\
d_{1}(u, v):=\int D(u, v)(t) d t, \quad d_{1}(u, V):=\int D(u, V)(t) d t .
\end{gathered}
$$

Then there exists a measurable multifunction $\Gamma: I \rightarrow \mathbb{R}^{n}$ with closed values such that $\Gamma(t)=\{v(t): v \in V\}$. Moreover, there exists a measurable selection $\gamma$ from $\Gamma$ such that

$$
d(u(t), \Gamma(t))=|u(t)-\gamma(t)|, \quad D(u, V)=D(u, \gamma), \quad d_{1}(u, V)=d_{1}(u, \gamma) .
$$

Proof. The existence of $\Gamma$ follows from Hiai-Umegaki [11]. For the existence of $\gamma$, see [2] or [12]. Finally, it is clear that if $v \in V$ then $v(t) \in \Gamma(t)$ a.e., hence $|u(t)-v(t)| \geq d(u(t), \Gamma(t))=|u(t)-\gamma(t)|$, i.e. $D(u, V) \geq D(u, \gamma)$ a.e. Since the opposite inequality is obvious, the equality holds.

Proposition 3. Let $G$ satisfy hypothesis $(\mathrm{G})$ and fix some $\left(u_{0}, v_{0}\right) \in$ $\operatorname{graph} G$. Then there exists a continuous map $\varrho_{u_{0} v_{0}}: K \rightarrow L_{+}^{1}$ such that

$$
\varrho_{u_{0}, v_{0}}\left(u_{0}\right)=0, \quad D\left(v_{0}, G(u)\right) \leq \varrho_{u_{0} v_{0}}(u) \quad \forall u \in K .
$$

Proof. See Fryszkowski [9, Proposition 2.2, Lemma 3.1] or BressanColombo [5, Propositions 4 and 5].

To simplify the statement of the next proposition, we define a set $\Lambda^{m} \subset$ $L^{1}\left(I, \mathbb{R}^{m}\right)$ which represents a partition of $I$ into $m$ disjoint measurable subsets. Namely, we set

$$
\Lambda^{m}:=\left\{\lambda \in L^{1}\left(I, \mathbb{R}^{m}\right): \lambda_{i}(t) \in\{0,1\} \text { and } \sum_{i=1}^{m} \lambda_{i}(t)=1 \text { a.e. }\right\} .
$$

Proposition 4. Let $p: K \rightarrow[0,1]^{m}$ be a continuous partition of unity, let $\varphi: K \rightarrow L^{1}\left(I, \mathbb{R}^{l}\right)$ be a continuous map, and fix $\varepsilon>0$. Then there exists a continuous map $\lambda: K \rightarrow \Lambda^{m}$ satisfying: 
(i) $\int \lambda(u) d \tau=p(u) \cdot T$;

(ii) $\left|\int \lambda_{i}(u)(\tau) \varphi(u)(\tau) d \tau-p_{i}(u) \int \varphi(u)(\tau) d \tau\right| \leq \varepsilon / m$;

(iii) $p_{i}(u)=1 \Rightarrow \lambda_{i}(u) \equiv 1 ; p_{i}(u)=0 \Rightarrow \lambda_{i}(u) \equiv 0$, a.e. $\forall u \in K \forall i$.

Proof. See Fryszkowski [9, Proposition 1.2].

Lemma 1. Let $G$ satisfy hypothesis $(\mathrm{G})$. Then for each $\varepsilon>0$ there exists a continuous map $g: K \rightarrow L^{1}$ such that

$$
d_{1}(g(u), G(u)) \leq \varepsilon, \quad\left|\int_{0}^{t}\left(g(u)(\tau)-g_{*}(u)(\tau)\right) d \tau\right| \leq \varepsilon \quad \forall t \in I \forall u \in K .
$$

Pr o of. Using the integrable boundedness of $G$ we can find a partition of $I$ into subintervals $I_{j}=\left[t_{j-1}, t_{j}\right), j=1, \ldots, m_{1}$, such that

$$
\forall u \in K \forall v \in G(u), \quad\left|\int_{I_{j}} v d s\right| \leq \varepsilon / 4, \quad j=1, \ldots, m_{1} .
$$

Since $g_{*}$ is continuous on $K$, we can find $\varepsilon^{\prime}$ such that, denoting by $d$ the distance in $K$,

$$
u_{1}, u_{2} \in K, d\left(u_{1}, u_{2}\right)<\varepsilon^{\prime} \Rightarrow d_{1}\left(g_{*}\left(u_{1}\right), g_{*}\left(u_{2}\right)\right)<\varepsilon / 4 .
$$

Set $\varepsilon_{1}:=\frac{1}{4} \min \left\{\varepsilon, \varepsilon^{\prime}\right\}$, and:

$$
\begin{aligned}
V_{j}(u) & :=\left\{\left.v\right|_{I_{j}}: v \in G(u), \quad \int_{I_{j}}\left(g_{*}(u)-v\right) d s=0\right\}, \quad j=1, \ldots, m_{1}, \\
V(u) & :=\left\{v \in G(u):\left.v\right|_{I_{j}} \in V_{j}(u), \forall j=1, \ldots, m_{1}\right\} .
\end{aligned}
$$

By Lyapunov's theorem on the range of vector measures (see [9]), $V_{j}(u)$ is nonempty $\forall j$, and since $G(u)$ is decomposable, we have $V(u) \neq \emptyset, \forall u \in K$. If we fix some $u_{0} \in K$ and some $v_{0} \in V\left(u_{0}\right)$ then by Proposition 3 there exists a continuous map $\varrho_{u_{0} v_{0}}$ such that $\varrho_{u_{0} v_{0}}\left(u_{0}\right)=0$ and $D\left(v_{0}, G(u)\right) \leq \varrho_{u_{0} v_{0}}(u)$, $\forall u \in K$; therefore the set

$$
U\left(u_{0}, v_{0}\right):=\left\{u \in K: d\left(u, u_{0}\right)<\varepsilon_{1},\left|\varrho_{u_{0} v_{0}}(u)\right|_{1}<\varepsilon_{1}\right\}
$$

is an open nbd of $u_{0}$. By compactness of $K$, the open cover $\left\{U\left(u_{0}, v_{0}\right): u_{0} \in\right.$ $\left.K, v_{0} \in V\left(u_{0}\right)\right\}$ has a finite subcover $\left\{U_{1}, \ldots, U_{m}\right\}$, where $U_{i}=U\left(u_{i}, v_{i}\right)$, and:

$$
\begin{gathered}
u \in U_{i} \Rightarrow d\left(u, u_{i}\right)<\varepsilon_{1}, d_{1}\left(g_{*}(u), g_{*}\left(u_{i}\right)\right)<\varepsilon / 4, \\
v_{i} \in G\left(u_{i}\right), \quad D\left(v_{i}, G(u)\right) \leq \varrho_{i}(u):=\varrho_{u_{i} v_{i}}(u), \quad\left|\varrho_{i}(u)\right|_{1}<\varepsilon_{1}, \\
\sum_{j=1}^{m_{1}}\left|\int_{I_{j}}\left(g_{*}(u)-v_{i}\right) d s\right| \leq d_{1}\left(g_{*}(u), g_{*}\left(u_{i}\right)\right)+\sum_{j=1}^{m_{1}}\left|\int_{I_{j}}\left(g_{*}\left(u_{i}\right)-v_{i}\right) d s\right|<\varepsilon / 4
\end{gathered}
$$


for $i=1, \ldots, m$. Let $p: K \rightarrow[0,1]^{m}$ be a subordinate continuous partition of unity, and apply Proposition 4 to $\varphi=\left(\varphi_{1}, \ldots, \varphi_{m+m m_{1} n}\right)$ defined by

$$
\varphi_{i}=\varrho_{i}:=\varrho_{u_{i} v_{i}}, \quad \varphi_{m+k}(u)(t)=\chi_{I_{j}}(t)\left[g_{*}(u)(t)-v_{i}(t)\right]_{r},
$$

for $i=1, \ldots, m, j=1, \ldots, m_{1}, r=1, \ldots, n, k=1, \ldots, m m_{1} n$, where $[\cdot]_{r}$ denotes the $r$ th component of the vector [.], with $\varepsilon_{1} / m_{1}$ in place of $\varepsilon$, obtaining a continuous map $\lambda: K \rightarrow \Lambda^{m}$ satisfying

$$
\begin{gathered}
\int \lambda(u) d \tau=p(u) \cdot T \\
\int \lambda_{i}(u) D\left(v_{i}, G(u)\right)(\tau) d \tau \leq p_{i}(u)\left|\varrho_{i}(u)\right|_{1}+\varepsilon_{1} / m m_{1} \leq\left(p_{i}(u)+1 / m\right) \varepsilon / 4 ; \\
\left|\int_{0}^{t_{j}} \lambda_{i}(u)\left(g_{*}(u)-v_{i}\right) d s\right| \leq \sum_{j=1}^{m_{1}}\left(p_{i}(u)\left|\int_{I_{j}}\left(g_{*}(u)-v_{i}\right) d s\right|+\varepsilon_{1} / m m_{1}\right) \\
\leq\left(p_{i}(u)+1 / m\right) \varepsilon / 4 \\
p_{i}(u)=1 \Rightarrow \lambda_{i}(u) \equiv 1 ; \quad p_{i}(u)=0 \Rightarrow \lambda_{i}(u) \equiv 0, \\
\text { a.e. } \forall u \in K \text { for } i=1, \ldots, m .
\end{gathered}
$$

Define now $g: K \rightarrow L^{1}, g(u):=\sum_{i=1}^{m} \lambda_{i}(u) v_{i}$. To see that $g$ is continuous, it is enough to note that

$$
\begin{aligned}
|g(u)-g(\underline{u})|_{1} & \leq \sum_{i=1}^{m} \int\left|\lambda_{i}(u)-\lambda_{i}(\underline{u})\right|\left|v_{i}\right| d s \\
& \leq \sum_{i=1}^{m} \int\left|\lambda_{i}(u)-\lambda_{i}(\underline{u})\right| M(s) d s,
\end{aligned}
$$

and each term in this sum is the integral of $M$ over a set of measure $\int \mid \lambda_{i}(u)-$ $\lambda_{i}(\underline{u}) \mid d s$, which clearly tends to 0 as $u \rightarrow \underline{u}$, since $\lambda_{i}: K \rightarrow L^{1}$ is continuous, $\forall j$. Moreover,

$$
\begin{aligned}
\left|\int_{0}^{t}\left(g_{*}(u)-g(u)\right) d s\right| \leq & \left|\int_{t_{j(t)}}^{t} g_{*}(u) d s\right|+\left|\int_{t_{j(t)}}^{t} g(u) d s\right| \\
& +\sum_{i=1}^{m}\left|\int_{0}^{t_{j(t)}} \lambda_{i}(u)\left[g_{*}(u)-v_{i}\right] d s\right| \\
\leq & \frac{1}{4} \varepsilon+\frac{1}{4} \varepsilon+\frac{1}{4} \varepsilon \sum_{i=1}^{m}\left(p_{i}(u)+1 / m\right)=\varepsilon .
\end{aligned}
$$

To see that $g$ is an $\varepsilon$-approximate selection from $G$, recall that by Proposition $2, \forall u \in K \exists v_{i}(u) \in G(u)$ such that $D\left(v_{i}, v_{i}(u)\right)=D\left(v_{i}, G(u)\right), i=1, \ldots, m$, 
so that, setting $v(u):=\sum_{i=1}^{m} \lambda_{i}(u) v_{i}(u) \in G(u), \forall u \in K$, we have

$$
\begin{aligned}
D(g(u), G(u)) \leq D(g(u), v(u)) & =\sum_{i=1}^{m} \lambda_{i}(u) D\left(v_{i}, v_{i}(u)\right) \\
& =\sum_{i=1}^{m} \lambda_{i}(u) D\left(v_{i}, G(u)\right) .
\end{aligned}
$$

Therefore

$$
d_{1}(g(u), G(u)) \leq \frac{1}{4} \varepsilon \sum_{i=1}^{m}\left[p_{i}(u)+1 / m\right] \leq \varepsilon \quad \forall u \in K .
$$

Lemma 2. Let $G$ satisfy hypothesis (G). Let $g^{k-1}: K \rightarrow L^{1}$ be a continuous map satisfying $d_{1}\left(g^{k-1}(u), G(u)\right) \leq \varepsilon_{k-1}$ for some $\varepsilon_{k-1}>0$. Then for any $0<\varepsilon_{k}<\varepsilon_{k-1}$ there exists a continuous map $g^{k}: K \rightarrow L^{1}$ such that

$$
d_{1}\left(g^{k}(u), G(u)\right) \leq \varepsilon_{k}, \quad d_{1}\left(g^{k}(u), g^{k-1}(u)\right) \leq \varepsilon_{k}+\varepsilon_{k-1} .
$$

Proof. Since $g^{k-1}$ is continuous on $K$, we can find $\varepsilon^{\prime}$ such that

$$
u, \underline{u} \in K, d(u, \underline{u})<\varepsilon^{\prime} \Rightarrow d_{1}\left(g^{k-1}(u), g^{k-1}(\underline{u})\right) \leq \varepsilon_{k} / 2 .
$$

Set $\varepsilon=\frac{1}{2} \min \left\{\varepsilon_{k}, \varepsilon^{\prime}\right\}$ and $V(u):=\left\{v \in G(u): d_{1}\left(g^{k-1}(u), v\right)=d_{1}\left(g^{k-1}(u)\right.\right.$, $G(u))\}$; then, by Proposition 2, $V(u) \neq \emptyset, \forall u \in K$. As in Lemma 1, for each $u_{0} \in K$ and each $v_{0} \in V\left(u_{0}\right)$, the set

$$
U\left(u_{0}, v_{0}\right)=\left\{u \in K: d\left(u, u_{0}\right)<\varepsilon,\left|\varrho_{u_{0} v_{0}}(u)\right|_{1}<\varepsilon\right\}
$$

is an open nbd of $u_{0}$, and the rest of the proof follows the steps of the proof of Lemma 1.

Proof of Theorem 1. Choose a positive decreasing sequence $\left(\varepsilon_{k}\right)$ such that $\sum \varepsilon_{k}=1 /(2 i)$, and apply Lemma 1 with $\varepsilon_{0}$ replacing $\varepsilon$, obtaining a continuous map $g^{0}: K \rightarrow L^{1}$ such that

$$
d_{1}\left(g^{0}(u), G(u)\right) \leq \varepsilon_{0}, \quad\left|\int_{0}^{t}\left(g_{*}(u)-g^{0}(u)\right) d s\right| \leq \varepsilon_{0}
$$

$\forall t \in I, \forall u \in K$. For $k=1,2, \ldots$, apply Lemma 2, obtaining a continuous $g^{k}: K \rightarrow L^{1}$ such that

$$
d_{1}\left(g^{k}(u), G(u)\right) \leq \varepsilon_{k}, \quad d_{1}\left(g^{k}(u), g^{k-1}(u)\right) \leq \varepsilon_{k}+\varepsilon_{k-1} .
$$

In particular, the sequence $\left(g^{k}(u)\right)$ is Cauchy, uniformly in $u \in K$, i.e. the sequence $\left(g^{k}\right)$ is a Cauchy sequence of continuous maps converging uniformly to some continuous $g_{1}: K \rightarrow L^{1}$ satisfying

$$
\begin{aligned}
d_{1}\left(g_{1}(u), G(u)\right) & \leq d_{1}\left(g_{1}(u), g^{k}(u)\right)+d_{1}\left(g^{k}(u), G(u)\right) \\
& \leq d_{1}\left(g_{1}(u), g^{k}(u)\right)+\varepsilon_{k} \rightarrow 0
\end{aligned}
$$


as $k \rightarrow \infty$, hence $g_{1}(u) \in G(u), \forall u \in K$. This means that $g_{1}$ is a continuous selection from $G$, and

$$
\begin{aligned}
&\left|\int_{0}^{t}\left[g_{*}(u)-g_{1}(u)\right] d s\right| \leq\left|\int_{0}^{t}\left[g_{*}(u)-g^{0}(u)\right] d s\right|+d_{1}\left(g^{0}(u), g^{1}(u)\right)+\ldots+ \\
&+d_{1}\left(g^{k-1}(u), g^{k}(u)\right)+d_{1}\left(g^{k}(u), g_{1}(u)\right) \\
& \leq \varepsilon_{0}+\left(\varepsilon_{0}+\varepsilon_{1}\right)+\ldots+\left(\varepsilon_{k-1}+\varepsilon_{k}\right)+d\left(g^{k}(u), g_{1}(u)\right) \\
& \rightarrow 2 \sum \varepsilon_{k}=1 / i \quad \forall t \in I \forall u \in K .
\end{aligned}
$$

Application to differential inclusions. Let $I$ be the interval $[0, T]$, let $\Xi$ be a compact convex set in $\mathbb{R}^{n}$ and $\Omega$ an open or closed set in $\mathbb{R}^{n}$.

Hypothesis (F). $F: I \times \Omega \rightarrow \mathbb{R}^{n}$ is a multifunction such that:

$\left(\mathrm{a}^{\prime}\right)$ the values $F(t, x)$ are compact;

(b') $\exists I_{0} \subset I$ such that $I \backslash I_{0}$ is a null set and $\left.F\right|_{I_{0} \times \Omega}$ is $\mathcal{L} \otimes \mathcal{B}$-measurable;

$\left(\mathrm{c}^{\prime}\right) \exists M: I \rightarrow \mathbb{R}^{+}$integrable such that: $y \in F(t, x) \Rightarrow|y| \leq M(t)$ a.e. and $d(y, \Xi) \leq|M|_{1} \Rightarrow y \in \Omega$

$\left(\mathrm{d}^{\prime}\right) F(t, \cdot)$ is lsc.

COROLlary 1. Let $F$ satisfy hypothesis $(\mathrm{F})$. Let $\left(\xi_{i}\right)$ be a sequence converging to some $\xi_{*}$ in $\Xi$. Let $f(t, x)$ be a selection from $\operatorname{co} F(t, x)$ which is measurable in $t$ and continuous in $x$. Then there exists a solution $x_{*}$ of $x^{\prime}=f(t, x), x(0)=\xi_{*}$, and a sequence $\left(x_{i}\right)$ of solutions of $(\mathrm{CP})$ with $x_{i}(0)=\xi_{i}$ such that $\left(x_{i}\right)$ converges uniformly to $x_{*}$.

Consider the compact convex subset of $C^{0}$ defined by

$$
K_{\infty}:=\left\{x \in C^{0}: x \in \mathrm{AC}, x(0) \in \Xi,\left|x^{\prime}(t)\right| \leq M(t) \text { a.e. }\right\} .
$$

Lemma 3. Let $F$ satisfy hypothesis $(\mathrm{F})$. Let $f(t, x)$ be a selection from co $F(t, x)$, measurable in $t$ and continuous in $x$. Then the function $g_{*}$ : $K_{\infty} \rightarrow L^{1}$ and the multifunction $G: K_{\infty} \rightarrow L^{1}$ defined by

$$
g_{*}(x)(t):=f(t, x(t)), \quad G(x):=\left\{v \in L^{1}: v(t) \in F(t, x(t)) \text { a.e. }\right\}
$$

satisfy hypothesis $(\mathrm{G})$.

Proof. Using the results of Hiai-Umegaki [11], it is clear that we need only prove that $G$ is lsc. To prove this notice first that for each $u \in K_{\infty}$ the multifunction $\Phi(t):=F(t, u(t))$ is measurable. In fact, for each closed set $C$ in $\mathbb{R}^{n}$ we can write

$$
\begin{aligned}
\Phi^{-}(C) & =\{t \in I: F(t, u(t)) \cap C \neq \emptyset\} \\
& =\{t \in I: F(t, \xi) \cap C \neq \emptyset \text { for some } \xi \text { with }(t, \xi) \in \operatorname{graph}(u)\} \\
& =\text { projection of } F^{-}(C) \cap \operatorname{graph}(u) \text { on } I .
\end{aligned}
$$


But, apart from a null set, this is the projection of an $\mathcal{L} \otimes \mathcal{B}$-measurable set, hence is measurable. Let $C$ be a closed set in $L^{1}$, and consider a sequence $\left(u_{k}\right) \rightarrow u_{0}$ such that $G\left(u_{k}\right) \subset C, \forall k \in \mathbb{N}$. Fix any $v_{0} \in G\left(u_{0}\right)$; since $G\left(u_{k}\right)$ is closed decomposable, by Proposition 2 there exists $v_{k} \in G\left(u_{k}\right)$ such that $D\left(v_{0}, v_{k}\right)=D\left(v_{0}, G\left(u_{k}\right)\right)$, hence for a.e. $t$ we have

$$
\left|v_{0}(t)-v_{k}(t)\right|=D\left(v_{0}, v_{k}\right)(t)=D\left(v_{0}, G\left(u_{k}\right)\right)(t)=d\left(v_{0}(t), F\left(t, u_{k}(t)\right)\right) ;
$$

but $F(t, \cdot)$ is lsc, $\left(u_{k}(t)\right) \rightarrow u_{0}(t)$, and $v_{0}(t) \in F\left(t, u_{0}(t)\right)$, hence $\mid v_{0}(t)-$ $v_{k}(t) \mid \rightarrow 0$ as $k \rightarrow \infty$. This means that $d_{1}\left(v_{0}, v_{k}\right) \rightarrow 0$, and since $\left(v_{k}\right) \subset C$, we have $v_{0} \in C$.

Proof of Corollary 1. Define $g_{*}$ and $G$ as in Lemma 3 . Then by Theorem 1 there exists a sequence $\left(g_{i}\right)$ of continuous selections from the multivalued Nemytskiı operator $G$ associated to $F$ such that, setting

$$
\begin{aligned}
h_{i}, h_{*}: K_{\infty} \rightarrow K_{\infty}, \quad & h_{i}(x)(t)=\xi_{i}+\int_{0}^{t} g_{i}(x)(\tau) d \tau, \\
h_{*}(x)(t) & =\xi_{*}+\int_{0}^{t} g_{*}(x)(\tau) d t,
\end{aligned}
$$

we have $\left(h_{i}\right) \rightarrow h_{*}$ uniformly.

It is clear that $h_{i}\left(K_{\infty}\right) \subset K_{\infty}$, and that $h_{i}$ is continuous. By the Schauder theorem, for each $i \in \mathbb{N}$ there exists a fixed point $x_{i}=h_{i}\left(x_{i}\right)$, i.e. $x_{i}^{\prime}=g_{i}\left(x_{i}\right) \in G\left(x_{i}\right), x_{i}(0)=\xi_{i}$. This means that $x_{i}^{\prime}(t) \in F(t, x(t))$ a.e. Since $\left(x_{i}\right)$ is a sequence in the compact $K_{\infty}$, a subsequence, which we denote again by $\left(x_{i}\right)$, converges to some $x_{*}$. It is clear that $x_{*}=h_{*}\left(x_{*}\right)$, so that $x_{*}^{\prime}(t)=f\left(t, x_{*}(t)\right)$ a.e.

Hypothesis (K). $F: I \times \mathbb{R}^{n} \rightarrow \mathbb{R}^{n}$ is a multifunction such that:

(a) the values $F(t, x)$ are compact;

(b) $F(\cdot, x)$ is measurable;

(c) $\exists M: I \rightarrow \mathbb{R}^{+}$integrable such that: $y \in F(t, x) \Rightarrow|y| \leq M(t)$ for a.e. $t$;

(d) $\exists w: I \times \mathbb{R}^{+} \rightarrow \mathbb{R}^{+}$such that $d(F(t, x), F(t, \underline{x})) \leq w(t,|x-\underline{x}|)$, with $w(\cdot, r)$ measurable, $w(t, \cdot)$ continuous concave, $w(t, 0)=0$ and $w(t, r)$ $\leq 2 M(t)$, for a.e. $t \in I$;

(e) the differential equation $r^{\prime}(t)=12 n w(t, r), r(0)=0$, has a unique AC solution on $[0, \underline{t}]$, for each $\underline{t}$ in $[0, T]$.

Condition (f). $f_{*}: I \times \mathbb{R}^{n} \rightarrow \mathbb{R}^{n}$ is a function such that:

$\left(\mathrm{b}^{\prime}\right) f_{*}(\cdot, x)$ is measurable;

(c') $\exists M$ as in (c) such that $\left|f_{*}(t, x)\right| \leq M(t)$ for a.e. $t$; 
(d $\mathrm{d}^{\prime} \exists w$ as in (d), (e) of hypothesis (K) such that $\left|f_{*}(t, x)-f_{*}(t, \underline{x})\right| \leq$ $12 n w(t,|x-\underline{x}|)$ for a.e. $t$.

COROLLARY 2. Let $F$ satisfy hypothesis $(\mathrm{K})$. Then for each solution $x_{*}$ of the relaxed Cauchy problem (CPR) there exists a selection $f_{*}$ from co $F(t, x)$ satisfying condition (f) such that $x_{*}$ is the unique solution of the differential equation

$$
x^{\prime}=f_{*}(t, x), \quad x(0)=\xi .
$$

In particular, the solution set $S(\xi)$ of $(\mathrm{CP})$ is dense in the solution set of the relaxed Cauchy problem (CPR).

Pro of. As in [15, Theorem 1 and Proposition 3], we can find a function $f$ such that $f(t, x, B)=\operatorname{co} F(t, x), B$ the unit ball in $\mathbb{R}^{n}$; and $u_{*}: I \rightarrow B$ such that $f\left(t, x_{*}(t), u_{*}(t)\right)=x_{*}^{\prime}(t)$ a.e., in such a way that the function $f_{*}$ defined by $f_{*}(t, x):=f\left(t, x, u_{*}(t)\right)$ satisfies condition (f). Now apply Corollary 1 and notice that (e) of hypothesis (K) holds.

Acknowledgement. I wish to thank Professor Arrigo Cellina for suggesting the problem and for useful discussions.

Remark. The referee pointed out the following:

(a) the interval $[0, T]$ can be replaced by a general separable measure space, just by using an isomorphism theorem, in the selection theorem;

(b) the $\delta_{0}$ constructed in Proposition 1 is usually called "ess inf $\Delta$ ";

(c) an interesting consequence of Theorem 1 is that the set of continuous selections from the multifunction $G$ is weakly dense in the set of continuous selections from $\overline{\mathrm{co}} G$, i.e. for every $g_{*}$ there exists a sequence $\left(g_{i}\right)$ such that

$$
\int_{0}^{T} \phi g_{i}(u) d s \rightarrow \int_{0}^{T} \phi g_{*}(u) d s
$$

for every measurable bounded $\phi:[0, T] \rightarrow \mathbb{R}$ (indeed, by Theorem 1 this certainly holds when $\phi$ is piecewise constant).

\section{References}

[1] H. A. Antosiewicz and A. Cellina, Continuous selections and differential relations, J. Differential Equations 19 (1975), 386-398.

[2] J. P. Aubin and A. Cellina, Differential Inclusions, Springer, New York 1984

[3] A. Bressan, On differential relations with lower-continuous right-hand side, J. Differential Equations 37 (1980), 89-97.

[4] -, On a bang-bang principle for non-linear systems, Boll. Un. Mat. Ital. Suppl. Anal. Funz. Appl. 1980, 55-59.

[5] A. Bressan and G. Colombo, Extensions and selections of maps with decomposable values, Studia Math. 90 (1988), 69-86. 
[6] A. Cellina and M. V. Marchi, Non-convex perturbations of maximal monotone differential inclusions, Israel J. Math. 46 (1983), 1-11.

[7] G. Colombo, A. Fonda and A. Ornelas, Lower semicontinuous perturbations of maximal monotone differential inclusions, ibid. 61 (1988), 211-218.

[8] A. F. Filippov, Classical solutions of differential equations with multivalued right hand side, SIAM J. Control 5 (1967), 609-621.

[9] A. Fryszkowski, Continuous selections for a class of non-convex multivalued maps, Studia Math. 76 (1983), 163-174.

[10] -, Continuous selections of Aumann integrals, J. Math. Anal. Appl., to appear.

[11] F. Hiai and H. Umegaki, Integrals, conditional expectations, and martingales of multivalued functions, J. Multivariate Anal. 7 (1977), 149-182.

[12] C. J. Himmelberg, Measurable relations, Fund. Math. 87 (1975), 53-72.

13] S. Łojasiewicz jr., The existence of solutions for lower semicontinuous orientor fields, Bull. Acad. Polon. Sci. 28 (1980), 483-487.

[14] C. Olech, Decomposability as a substitute for convexity, in: Multifunctions and Integrands, G. Salinetti (ed.), Lecture Notes in Math. 1091, Springer, Berlin 1984, 193-205.

[15] A. Ornelas, Parametrization of Carathéodory multifunctions, preprint SISSA 51 M (1988).

[16] G. Pianigiani, On the fundamental theory of multivalued differential equations, J. Differential Equations 25 (1977), 30-38.

[17] A. Pliś, Trajectories and quasitrajectories of an orientor field, Bull. Acad. Polon. Sci. 11 (1963), 369-370.

[18] A. A. Tolstonogov and I. A. Finogenko, On solutions of a differential inclusion with lower semicontinuous nonconvex right-hand side in a Banach space, Math. USSR-Sb. 53 (1986), 203-231.

[19] T. Ważewski, Sur une généralisation de la notion des solutions d'une équation au contingent, Bull. Acad. Pol. Sci. 10 (1962), 11-15.

DEPARTAMENTO DE MATEMÁTICA

UNIVERSIDADE DE ÉVORA

APARTADO 94

7001 ÉVORA CODEX, PORTUGAL 